\title{
"How does Austria sleep?" self-reported sleep habits and complaints in an online survey
}

\author{
Christine Blume ${ }^{1,2,3}$ (D) Theresa Hauser ${ }^{4}$ - Walter R. Gruber ${ }^{3}$. Dominik PJ Heib ${ }^{3,4}$ - Thomas Winkler ${ }^{4}$. \\ Manuel Schabus ${ }^{3,4}$
}

Received: 1 July 2019 /Revised: 23 October 2019 / Accepted: 20 November 2019/Published online: 14 December 2019

(C) The Author(s) 2019

\begin{abstract}
During the past years, the prevalence of sleep problems has been increasing steadily in industrial societies and represents a major social and socioeconomic burden. The situation in Austria was last evaluated in 2007 by Zeitlhofer and colleagues in a representative sample of 1000 participants. In the current study, we sought to evaluate the sleep behaviour of the Austrian population in an ongoing online survey, in which we have collected data from 986 participants (66\% women, mean age $40.9 \pm 16.4$ years) between March 2018 and May 2019. Sleep duration was appropriate in 52\% of the respondents (i.e. 7-9 h per night). However, we found an alarmingly high number of self-reported sleep problems (46\%), and only $31 \%$ of the participants classified themselves as "good sleepers" using a validated self-report questionnaire (Pittsburgh Sleep Quality Index, PSQI). Furthermore, many participants reported suffering from sleep problems for a very long time $(86 \%>6$ months; $37 \%>5$ years) suggesting that currently available treatment options are either ineffective or not employed. Possible reasons for sleep problems could include irregular sleep-wake cycles, increased perceived stress levels, and the use of electronic devices just before sleep.
\end{abstract}

Keywords Sleep $\cdot$ Online survey $\cdot$ Austria $\cdot$ Sleep problems $\cdot$ Sleep duration $\cdot$ Sleep quality

\section{Introduction}

Especially in Western societies, an increasingly high number of people complain about sleep problems. Among clinically relevant sleep disorders, insomnia, which is characterized by problems initiating and maintaining sleep and the feeling of sleep not being restorative, is extremely

Electronic supplementary material The online version of this article (https://doi.org/10.1007/s11325-019-01982-5) contains supplementary material, which is available to authorized users.

Manuel Schabus

manuel.schabus@sbg.ac.at

1 Centre for Chronobiology, Psychiatric Hospital of the University of Basel, Basel, Switzerland

2 Transfaculty Research Platform Molecular and Cognitive Neurosciences, University of Basel, Basel, Switzerland

3 Centre for Cognitive Neuroscience, University of Salzburg, Salzburg, Austria

4 Laboratory for Sleep, Cognition, and Consciousness Research, University of Salzburg, Salzburg, Austria prevalent. Subjectively, patients suffer from significant distress or impairment in social, occupational or other important areas of daytime functioning. Globally, insomnia is the most commonly reported sleep problem in industrialized countries [1], and numbers seem to have been increasing steadily during the past years [2]. Epidemiological research suggests that its prevalence is between 10 and 35\% in the general adult population [3-6]. Besides subjective suffering, sleep problems are also associated with a significant economic burden resulting from high direct (i.e. due to health-care consultations and treatment) and indirect (i.e. due to absenteeism and loss of productivity) costs [7, 8]. For these numbers to be translated into adequate healthcare strategies, it is essential to know about the situation in a specific country. While a study from the Robert Koch Institute [9] presents data from 2010 on the situation among the adult population in Germany, the last appraisal from Austria dates back to 2007 [10]. However, especially given the reported increase in sleep problems during the last years, these numbers are likely to be outdated. Therefore, we opened a web-based survey in March 2018 to obtain a precise appraisal of the sleep quality and sleep 
habits of the Austrian population. We here present the results of the survey, in which 986 people had participated as of May 2019.

\section{Methods and materials}

\section{Survey}

The survey is accessible on www.sleeplounge.net and run on LimeSurvey [11], and informed consent was inherent to the study and covered by an ethical framework agreement from the University of Salzburg. The survey included two compulsory questionnaires, namely, (i) the Pittsburgh Sleep Quality Index [PSQI; 12] and (ii) a questionnaire to assess general health and sleep habits [adapted from 10]. The (iii) Morningness-Eveningness Questionnaire [D-MEQ; 13, 14] as well as a (iv) questionnaire on sleep problems and one on the (v) attitude regarding clock change and the use of electronic devices in the evenings were optional. As not all participants filled in all questionnaires, the sample size slightly varies between the different results reported below. Recruitment of respondents was via public relations activities of individual members of the laboratory as well as the University of Salzburg.

\section{Sample}

A total of 986 people (33.67\% men, $66.33 \%$ women) had participated in the survey on sleep habits as of May 15, 2019. While all participants lived in Austria, the sample comprised $88 \%$ Austrian citizens, and $12 \%$ were other nationalities. The sample comprised participants between 15 and 90 years with a mean age of 40.9 years ( \pm 16.4 years). About eighty-eight percent were between 18 and 64 years old [general population: 61.8\% according to data from Statistik Austria from 2018; 15], and $8.82 \%$ were older than 64 years [general population: $18.8 \%$ according to data from Statistik Austria from 2018; 15]. In general, a high proportion of participants (data were available from $N=717$ participants, who answered the questionnaire on sleep problems) held a university degree ( $50.63 \%$ compared to $20 \%$ in the general population between 25 and 64 [16]). In the survey part on sleep problems, 764 people had participated (35.60\% men, $64.40 \%$ women) with a mean age of 39.1 years ( \pm 16.2 years). For the specification of the chronotype, 611 (32.24\% men, $67.76 \%$ women) respondents had submitted their answers, a total of 267 (33.33\% men, 66.67\% women) respondents had participated in the clock change questionnaire, and 264 (33.33\% men, 66.67\% women) had participated in the part about the use of electronic devices before sleep. For more details on the sample, please see Table 1. Please note that although the survey sample may not be representative for the

Table 1 Sociodemographic data of all participants

\begin{tabular}{|c|c|c|c|c|c|c|c|}
\hline & & \multicolumn{2}{|l|}{ Total } & \multicolumn{2}{|c|}{ Male } & \multicolumn{2}{|c|}{ Female } \\
\hline & & $\mathrm{n}$ & $\%$ & $\mathrm{n}$ & $\%$ & $\mathrm{n}$ & $\%$ \\
\hline \multicolumn{2}{|l|}{ Total } & 986 & 100 & 332 & 33.7 & 654 & 66.3 \\
\hline \multirow[t]{5}{*}{ Age } & $<30$ & 332 & 33.7 & 83 & 25.0 & 249 & 38.1 \\
\hline & $30-45$ & 258 & 26.2 & 87 & 26.2 & 171 & 26.1 \\
\hline & $46-59$ & 248 & 25.2 & 89 & 26.8 & 159 & 24.3 \\
\hline & $>60$ & 148 & 15.0 & 73 & 22.0 & 75 & 11.5 \\
\hline & $\begin{array}{l}\text { Total } \\
\text { (missing) }\end{array}$ & $717(269)$ & 100 & & & & \\
\hline \multirow{6}{*}{$\begin{array}{l}\text { Highest educational } \\
\text { level obtained }\end{array}$} & None & 9 & 1.2 & 3 & 1.1 & 6 & 1.2 \\
\hline & Lower secondary school & 20 & 2.6 & 9 & 3.3 & 11 & 2.2 \\
\hline & Apprenticeship & 66 & 9.2 & 37 & 13.6 & 29 & 5.9 \\
\hline & Vocational school & 71 & 9.9 & 21 & 7.7 & 50 & 10.2 \\
\hline & Higher education & 188 & 26.2 & 70 & 25.7 & 118 & 24.0 \\
\hline & University degree & 363 & 50.6 & 117 & 43.0 & 246 & 50.0 \\
\hline \multirow[t]{6}{*}{ Occupation } & Unemployed & 14 & 1.8 & 4 & 1.5 & 10 & 2.0 \\
\hline & Retired & 75 & 9.8 & 33 & 12.1 & 42 & 8.5 \\
\hline & Student & 230 & 30.1 & 74 & 27.2 & 156 & 31.7 \\
\hline & Freelancer & 40 & 5.2 & 17 & 6.3 & 23 & 4.7 \\
\hline & Worker & 13 & 1.7 & 9 & 3.3 & 4 & 0.8 \\
\hline & Employee & 392 & 51.3 & 135 & 49.6 & 257 & 52.2 \\
\hline
\end{tabular}

Age distributions are from the sleep habits questionnaire $(N=986)$, and educational levels as well as information on occupation are from the sleep problems questionnaire ( $n=717$ of 764 gave information on their occupation) 
Austrian population, representativeness is difficult to obtain in web-based surveys due to self-selection.

\section{Statistical evaluation}

For between-group comparisons, a Welch two sample $t$-test was computed. For correlations between two ordinal variables, we report Kendall's tau, for the correlation between two interval scale variables a Pearson correlation, and for the correlation between an interval scale variable and a dichotomous variable the point biserial correlation. For the analyses of the relationships between two dichotomous variables from fourfold tables, we report results from Chi-square tests. Statistical evaluation was done in $\mathrm{R}$ version 3.6.1 [17]. We report $p$ values for statistical tests, all tests were two-sided.

\section{Results}

\section{Sleep duration, quality and chronotype}

About $52 \%$ of the respondents slept between 7 and $9 \mathrm{~h}$ per night (cf. Supplementary Tables S1 and S2 for details), and the mean sleep duration was $6.7 \mathrm{~h}( \pm 1.2 \mathrm{~h})$. About $47 \%$ were reported that they usually sleep less than $7 \mathrm{~h}$. Here, university students or academics slept, on average, longer than nonacademics $(6.97 \pm 1.14 \mathrm{~h}$ vs. $6.26 \pm 1.21 \mathrm{~h} ; t(510.47)=7.80$, $p<.001)$. Approximately $89 \%$ of those who took the German version of the Morningness-Eveningness Questionnaire $(n=$ 197 men and $n=414$ women) were neutral types, moderate evening or moderate morning types, $8.67 \%$ were definite morning and $2.26 \%$ definite evening types (cf. Supplementary Table S3 for details). Among respondents reporting (very) poor sleep quality, a disproportionately high number seemed to sleep less than $7 \mathrm{~h}$ (cf. Figure 1 and Supplementary Table S4 for details). This is confirmed by a correlation between sleep quality and sleep duration $(\operatorname{tau}=.39, p<.001)$.

\section{Sleep problems}

A total of $45.84 \%$ ( $n=452$ of 986 participants) indicated they had sleep problems when they were asked whether they currently had a sleep problem. About $40 \%$ (of $n=764$ participants) moreover reported (very) poor sleep when asked to rate their sleep quality on a four-step Likert scale (i.e. "very good", "good", "poor" or "very poor"). While the prevalence of selfreported sleep problems generally increased with age, their prevalence was higher for women aged 60 or older compared to men $\left(\chi^{2}(1)=4.09, p=.043\right.$; cf. Figure 2A and Suppl. Table S5). Among those with self-reported sleep problems, $52.65 \%$ (55.70\% men vs. $51.16 \%$ women, $24.14 \%$ of all participants) indicated they suffered from early awakenings, $70.35 \%(62.42 \%$ men vs. $74.26 \%$ women, $32.25 \%$ of all
Sleep Quality \& Sleep Duration

$(N=764)$

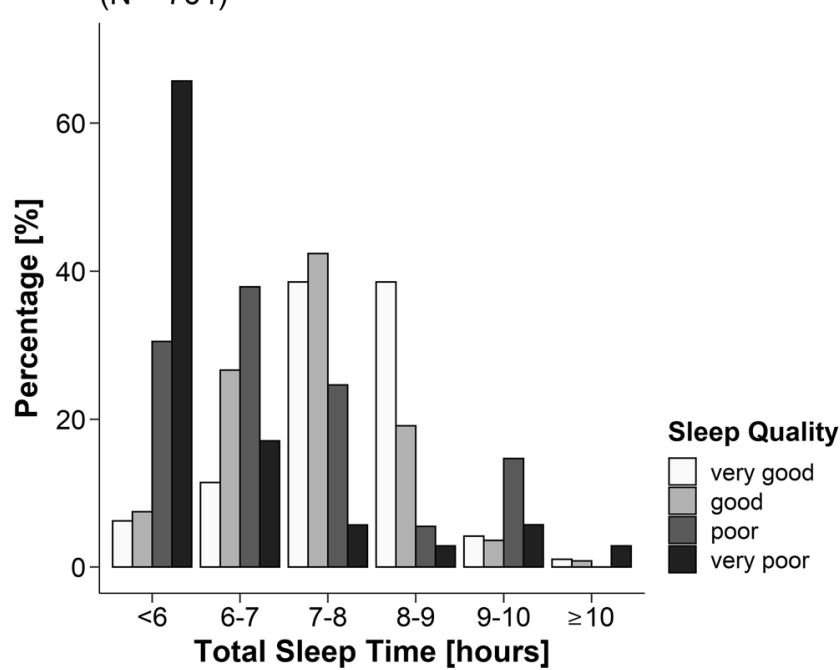

Fig. 1 Total sleep time and self-reported sleep quality during the past 4 weeks. About half of the participants report sleeping between 7 and $9 \mathrm{~h}$ per night. Participants with worse sleep quality more often report a shorter sleep duration. Percentages are scaled so participants of each sleep quality category sum up to $100 \%$. Note that numbers on the $x$-axis refer to, e.g. 6-6.99 h

participants) said they had problems maintaining sleep, and $50.44 \%$ (47.65\% of men vs. $51.81 \%$ women, $23.12 \%$ of all participants) reported problems falling asleep. About $11 \%$ reported that they were currently using sleep medication and $6.08 \%$ indicated they had used it in the past. About $86 \%$ indicated that their sleep problems had existed for more than 6 months, $37.17 \%$ for more than 5 years (cf. Supplementary Table S6), and only $5.31 \%$ had been suffering for less than 6 months. About $57 \%$ of all participants felt generally burdened at the time of assessment with women generally feeling more burdened than men $\left(\chi^{2}(1)=4.48, p=.034\right.$; cf. Supplementary Table S7). Of those who felt burdened, $49.90 \%$ indicated this was due to daily demands at work or university and $22.81 \%$ due to multiple burdens. Other reasons were psychological or physical strains $(12.22 \%)$ and familyor partnership-related issues (10.18\%).

About 14\% reported that they had (almost) had an accident due to fatigue with the nature of the accident not being detailed. About $11 \%$ of the respondents reported unintended napping during the day, and $18.45 \%$ reported intended habitual naps. Also, only in the age group below 30 $\left(\chi^{2}(1)=7.89, p=.005\right)$, sleep problems were more prevalent among participants with irregular sleep-wake cycles (i.e. not the same sleep times every day) than regular ones (cf. Figure 2C and Supplementary Table S8 for details). Moreover, regularity of sleep-wake cycles increased with age (i.e. $<30$ years: $55.74 \%$ irregular vs. $44.26 \%$ regular; 30-44.99 years: $33.62 \%$ irregular vs. $66.38 \%$ regular; $45-$ 59.99 years: $34.02 \%$ irregular vs. $65.98 \%$ regular; $\geq$ 

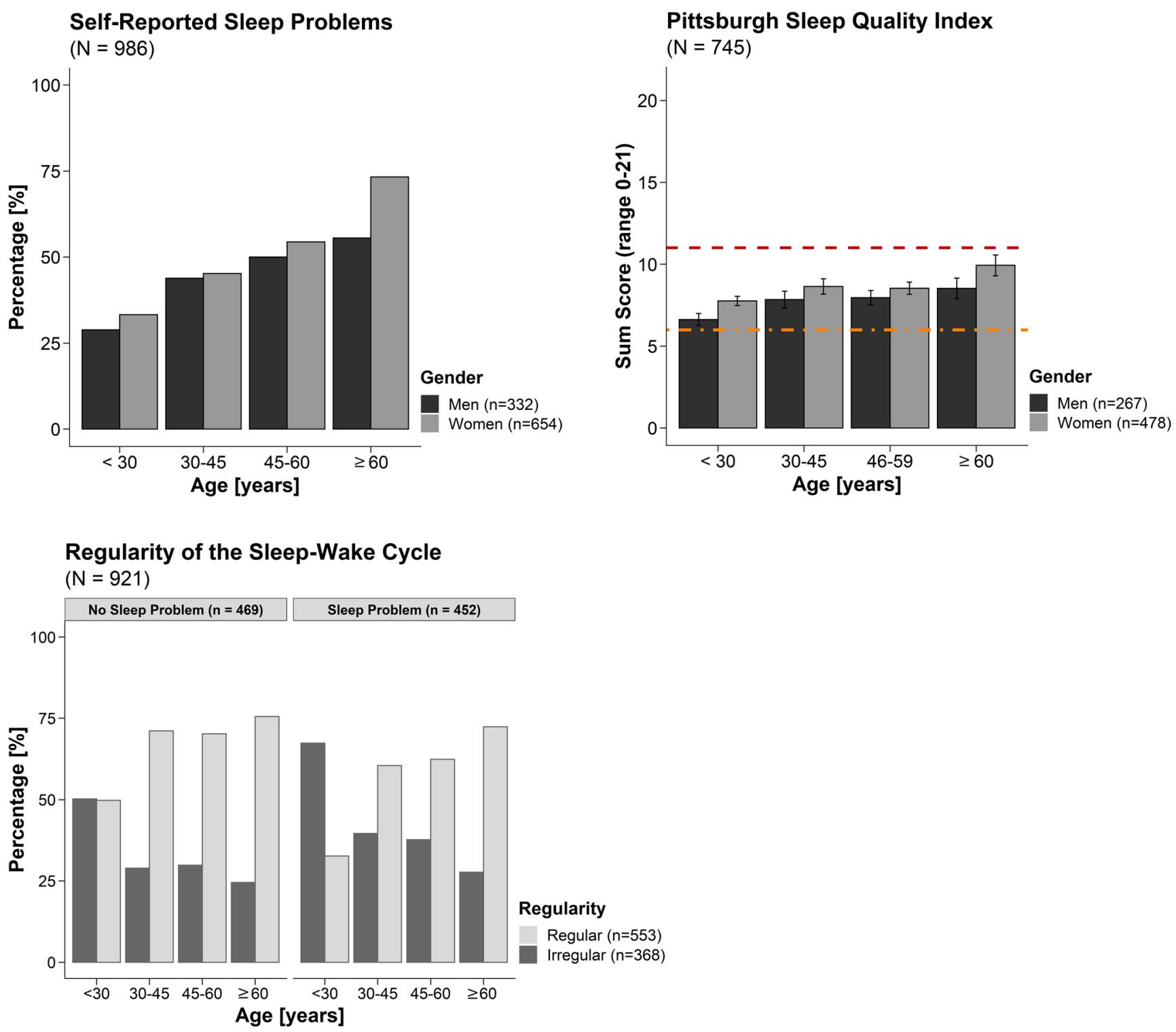

Fig. 2 (A) Percentages of participants reporting sleep problems according to gender and age group. In all age groups, women report more sleep problems than men, and the percentage of self-reported sleep problems increases considerably with age. (B) Sum scores of the Pittsburgh Sleep Quality Index [PSQI; 12] according to gender and age group. In all age groups and irrespective of gender, the average score was above 5 indicating suboptimal sleep quality. Women had slightly worse sleep quality than men, and overall, sleep quality decreased with increasing age. The dot-dashed orange line indicates the cut-off point between good and bad

60 years: $25.00 \%$ irregular vs. $75.00 \%$ regular). This effect is also statistically significant $\left(\chi^{2}(3)=49.75, p<.001\right)$.

\section{PSQI}

Results from the PSQI indicated that, on average, no age group irrespective of gender reported good sleep quality sleep quality (i.e. sum score $\geq 6$ ), and the dashed red line indicates the cutoff commonly chosen for severe sleep problems [i.e. sum score $\geq 11$; e.g. 18]. The PSQI sum score has a minimum of 0 (no sleep complaints) and a maximum of 21 (sleep disorder). (C) Percentages of participants with sleep problems reporting a regular vs. irregular sleep-wake cycle. Only in those younger than 30 sleep problems were more prevalent among those reporting irregular sleep-wake cycles. Please note that percentages are scaled, so participants of each age group with and without a regular sleep-wake cycle and self-reported sleep problems sum up to $100 \%$

(as indicated by a PSQI score $\leq 5$, cf. Figure 2B and Supplementary Table S9). Overall, only $31.28 \%$ of the respondents were "good sleepers", $41.07 \%$ were "poor sleepers" (PSQI score between 6 and 10), and 27.65\% had severe sleep problems [PSQI score > 10; e.g. 18] according to this self-evaluation tool. Generally, PSQI scores increased with age $(r=.17, p<.001)$, and women had higher PSQI values than men $(r=.084, p=.021)$. 


\section{Use of electronic devices before sleep}

When asked until when before switching off lights they used light-emitting electronic devices such as laptops, smart phones or tablets, $44.32 \%$ of 264 participants (176 women) said they used it until just before switching off the lights to sleep. About $19 \%$ switched it off between 5 and $10 \mathrm{~min}$ before sleep and $16.3 \%$ within $10-30 \mathrm{~min}$ before sleep. Only about $20 \%$ of the respondents indicated they do not use electronic devices within the hour before switching off lights (cf. Supplementary Table S10 for details).

\section{Clock change}

From 267 participants (178 women), 77.90\% indicated they did not at all or only slightly suffer from the spring clock change, for the change in autumn this was reported by $80.90 \%$. About $12 \%$ said they suffered somewhat during spring $(9.36 \%$ in autumn), and $10.11 \%$ said they suffered a lot or extremely following the spring clock change (likewise $9.74 \%$ in autumn). When asked how long they needed to adapt to the new time, $35.21 \%$ said they adapt immediately in spring (40.82\% in autumn), $44.57 \%$ said they adapt within $1-3$ days ( $40.45 \%$ in autumn), and only $20.22 \%$ said they need 4 or more days to adapt $(18.73 \%$ in autumn; cf. Supplementary Table S11 for details). About $58 \%$ of the participants would abolish clock change, and $57.79 \%$ of these would choose perennial daylight-saving time (or "summer" time) compared to $40.26 \%$ who would prefer standard time (or "winter" time) if they were to choose.

\section{Discussion}

The "How does Austria sleep?" survey provides important insights into sleep behaviour and sleep problems in a convenience sample from the Austrian population in 2018/2019. While general sleep parameters such as sleep duration generally seem to be in line with what has been reported in a previous study [10], we identify an alarmingly high number of participants with sleep problems in a relatively young sample with an above-average educational level. Moreover, these sleep problems are in many cases chronic suggesting that the available treatment options may not be adequate.

In more detail, in this online survey, $73.4 \%$ of the respondents reported an average sleep duration between 6 and $8 \mathrm{~h}$ per night and $52.4 \%$ between 7 and 9 h. However, $46.5 \%$ also report that they usually sleep less than $7 \mathrm{~h}$. This suggests that, in this sample, the average sleep duration is comparable to what has previously been reported by Zeitlhofer and colleagues in 2007 [10], although a substantial number of participants also does not sleep enough according to expert recommendations [19]. Generally, a sleep duration between 7 and
$9 \mathrm{~h}$ has been recommended for adults [19], while short $(<6 \mathrm{~h})$ and long ( $>9 \mathrm{~h}$ ) sleep has been associated with increased mortality and morbidity [20,21]. Although it is still unclear whether there might be a causal relationship between long sleep duration and mortality, it seems more likely that people who sleep unusually long suffer from chronic diseases or disorders such as sleep apnea resulting in non-restorative sleep [22-24].

Regarding sleep problems, $45.8 \%$ of the sample reported that they currently suffered from sleep problems. The results from the PSQI, a valid and reliable tool to identify sleep disorders, paint an even more alarming picture with only $31 \%$ of the participants being identified as "good sleepers", $41 \%$ as "poor sleepers" and $28 \%$ even reporting severe sleep problems. More specifically, $23 \%$ of the sample reported problems falling asleep, $24 \%$ indicated they suffered from early awakenings, and $32 \%$ had problems maintaining sleep. A recent but still unpublished study from the Medical University of Vienna [25] suggests a considerable increase in the prevalence of sleep problems compared to the data from 2007, when only $6 \%$ reported problems initiating sleep, $9 \%$ complained about early awakenings and $26 \%$ reported problems maintaining sleep [10]. Although the present sample cannot be compared to these findings directly, it should be noted that the sample in the present survey was rather young (average of 40.9 years; $59.5 \%<46$ years) and well-educated with a presumably good socioeconomic status $(51 \%$ academics, $26 \%$ higher education). This reflects a combination of characteristics that is usually associated with a decreased risk for sleep problems [26]. Besides this, in our sample, $86 \%$ of the respondents indicated they had been suffering from sleep problems for more than 6 months, and an unacceptably high number of $37 \%$ had even been suffering for more than 5 years. A possible reason for the high prevalence of sleep disorders could be the subjectively perceived burden. Indeed, approximately $57 \%$ of our sample felt burdened, and this was correlated with sleep problems (burdened "yes" vs. "no", sleep problems "yes" vs. "no"; $\left.\chi^{2}(1)=70.96, p<.001\right)$. The apparent focus on work-related demands may also have arisen from the fact that the sample was, on average, highly educated with $51 \%$ holding a university degree. More specifically, a high educational level often comes with more degrees of freedom and responsibility at work, which may increase perceived strain. For university students, several recent studies have emphasized that they are a highly burdened group at risk for (mental) health problems [27]. Another reason for sleep problems could also be irregular sleep-wake cycles. This effect was only statistically significant in young participants below the age of 30 though.

Besides sleep habits and sleep complaints, we also sought to investigate the use of electronic devices such as smart phones, laptops and e-readers before sleep. Here, $44 \%$ indicated they used a device until just before switching off the lights to sleep, and 20\% switched it off just between 5 and 
10 min before sleep. Critically, on the one hand, it has been suggested that the high proportions of short-wavelength light (i.e. in the "blue" range) of light-emitting diode (LED) screens may be detrimental for sleep by suppressing melatonin and increasing alertness [28-30]. On the other hand, it may also be psychological features such as the entertaining and emotionally arousing character of electronic devices that are responsible for the negative effects. The extent to which each of these factors contribute is still unknown.

Last, we were also interested in the opinion of the people living in Austria on clock change, which the EU parliament wishes to abolish in 2020. In our sample, 58\% would abolish clock change, and, of these, $57.8 \%$ would favour perennial daylight-saving or "summer" time (DST). While this slight preference for DST is in line with results from a nonrepresentative EU-wide survey from 2018 [31], the number of those in favour of abolishing clock change was considerably higher in the EU survey compared to ours (i.e. $84 \%$ in the EU survey vs. $58 \%$ in ours). This may be due to self-selection with a very specific survey such as the one initiated by the EU particularly attracting participants who have a strong opinion on a topic. Interestingly, when we asked how much they suffered from clock change, $76 \%$ of our respondents indicated they did not at all or only slightly suffer from clock change. About $69 \%$ said they adapt immediately (38\%) or within 1-2 days (31\%) to the new clock time, and another $21 \%$ said they adapt within 3-5 days. This is well in line with the normal adaptation times of the circadian system to trans meridian travel, i.e. one time zone per day as a rule of thumb [32], and suggests that clock change may be less of a burden for many people compared to the impression generated in public debates.

One limitation of the study is that the sample, in contrast to the study by Zeitlhofer and colleagues [10], was not a representative for the Austrian population. In particular, the educational level was above average and a disproportionately high number of women participated in the study. However, in online surveys, representativeness is more difficult to obtain than in studies using face-to-face interviews due to mechanisms of self-selection. This may, unintendedly, also have led to good sleepers generally being less interested in participating in the study resulting in an overrepresentation of participants with sleep problems. Therefore, current efforts aim at inviting currently underrepresented parts of the population to this ongoing survey, for example, in the context of public-relations activities of the Salzburg sleep laboratory and articles in the lay press.

In conclusion, sleep duration was sufficient in about 50\% of the respondents but insufficient (i.e. habitually less than $7 \mathrm{~h}$ ) in $47 \%$. Generally, sleep duration is therefore comparable to what has been reported in 2007 [10]. However, it is unclear to what extent this is a specific characteristic of the present sample. On a less positive note, the prevalence of sleep problems is alarmingly high, even in a sample that would usually be expected to be less prone to sleep problems. The persistence of sleep complaints for an unacceptably long time suggests that the current treatment options are either ineffective or, more probably, not used by patients. This may, for example, be due to limited capacities of sleep laboratories or patients not being satisfied with the options offered to them, which is often sleep medication, although the first-line treatment should be cognitive-behavioural therapy for insomnia [CBT-I; 33]. Other reasons may be that patients do not recognize their condition's pathological significance, or patients simply not knowing where to seek help. Besides increasing the availability of effective low-threshold therapeutic options, psychoeducational approaches should aim at increasing the sensitivity of the population regarding the detrimental effects of, for example, the use of electronic devices just before sleep.

Funding information Open access funding provided by Austrian Science Fund (FWF). C.B. is supported by an Erwin-Schroedinger-Fellowship from the Austrian Science Fund (FWF J-4243), a grant from the Freiwillige Akademische Gesellschaft (FAG) Basel and a grant from the Psychiatric Hospital of the University of Basel.

\section{Compliance with ethical standards}

Conflict of interest The authors declare that they have no conflict of interest.

Ethical approval All procedures performed in studies involving human participants were in accordance with the ethical standards of the institutional and/or national research committee and with the 1964 Helsinki declaration and its later amendments or comparable ethical standards. Informed consent was obtained from all individual participants included in the study.

Open Access This article is licensed under a Creative Commons Attribution 4.0 International License, which permits use, sharing, adaptation, distribution and reproduction in any medium or format, as long as you give appropriate credit to the original author(s) and the source, provide a link to the Creative Commons licence, and indicate if changes were made. The images or other third party material in this article are included in the article's Creative Commons licence, unless indicated otherwise in a credit line to the material. If material is not included in the article's Creative Commons licence and your intended use is not permitted by statutory regulation or exceeds the permitted use, you will need to obtain permission directly from the copyright holder. To view a copy of this licence, visit http://creativecommons.org/licenses/by/4.0/.

\section{References}

1. Sateia $\mathrm{M}$ et al (2000) Evaluation of chronic insomnia. an American Academy of sleep medicine review. Sleep 23(2):243-308

2. Beck F, Richard JB, Léger D (2013) Insomnia and total sleep time in France: prevalence and associated socio-demographic factors in a general population survey. Rev Neurol 169(12):956-964

3. Gallup-Organization, Sleep in America. 1995, Princeton (NJ): Gallup Organization

4. Angst, J., et al., The Zurich study .7. insomnia - symptoms, classification and prevalence. Eur Arch Psychiatry Clin Neurosci, 1989. 238(5-6): p. 285-293 
5. Morin CM, LeBlanc M, Daley M, Gregoire JP, Mérette C (2006) Epidemiology of insomnia: prevalence, self-help treatments, consultations, and determinants of help-seeking behaviors. Sleep Med 7(2):123-130

6. Johnson EO, Roth T, Schultz L, Breslau N (2006) Epidemiology of DSM-IV insomnia in adolescence: lifetime prevalence, chronicity, and an emergent gender difference. Pediatrics 117(2):E247-E256

7. Ozminkowski RJ, Wang S, Walsh JK (2007) The direct and indirect costs of untreated insomnia in adults in the United States. Sleep 30(3):263-273

8. Daley M et al (2009) The economic burden of insomnia: direct and indirect costs for individuals with insomnia syndrome, insomnia symptoms, and good sleepers. Sleep 32(1):55-64

9. Schlack, R., et al., Häufigkeit und Verteilung von Schlafproblemen und Insomnie in der deutschen Erwachsenenbevölkerung. 2013, Robert Koch-Institut, Epidemiologie und Gesundheitsberichterstattung

10. Zeitlhofer J et al (2010) Sleep habits and sleep complaints in Austria: current self-reported data on sleep behaviour, sleep disturbances and their treatment. Acta Neurol Scand 122(6):398-403

11. Limesurvey GH LimeSurvey: an open source survey tool. LimeSurvey GmbH: Hamburg. Germany

12. Buysse DJ, Reynolds CF 3rd, Monk TH, Berman SR, Kupfer DJ (1989) The Pittsburgh sleep quality index: a new instrument for psychiatric practice and research. Psychiatry Res 28(2):193-213

13. Griefahn B (2002) The validity of the temporal parameters of the daily rhythm of melatonin levels as an indicator of morningness. Chronobiol Int 19(3):561-577

14. Griefahn B et al (2001) Zur Validität der deutschen Übersetzung des Morningness-Eveningness-questionnaires von Horne und Östberg: the validity of a German version of the Morningness-Eveningnessquestionnaire developed by Horne and Östberg. Somnologie 5(2): $71-80$

15. Statistik AUSTRIA. Bevölkerung. 2019 [cited 2019 15-05]; Available from: http://www.statistik.at/web_de/statistiken/ menschen_und_gesellschaft/bevoelkerung/index.html

16. OECD. Education at a Glance: OECD Indicators (Austria). 2014 [cited 2019 15-05]; Available from: https://www.oecd.org/ education/Austria-EAG2014-Country-Note.pdf

17. R Core Team, $R$ : a language and environment for statistical computing. 2015, R Foundation for statistical computing: Vienna, Austria

18. Schlarb AA et al (2017) Sleep disturbances and mental strain in university students: results from an online survey in Luxembourg and Germany. Int J Ment Heal Syst 11:24-24

19. Consensus Conference Panel, et al., Joint consensus statement of the American Academy of Sleep Medicine and Sleep Research Society on the recommended amount of sleep for a healthy adult: methodology and discussion. Sleep, 2015. 38(8): p. 1161-1183
20. Itani O, Jike M, Watanabe N, Kaneita Y (2017) Short sleep duration and health outcomes: a systematic review, meta-analysis, and metaregression. Sleep Med 32:246-256

21. Jike M et al (2018) Long sleep duration and health outcomes: a systematic review, meta-analysis and meta-regression. Sleep Med Rev 39:25-36

22. Liu Y et al (2018) Relationship between sleep duration and selfreported health-related quality of life among US adults with or without major chronic diseases, 2014. Sleep Health: Journal of the National Sleep Foundation 4(3):265-272

23. Roure N, Gomez S, Mediano O, Duran J, Peña Mde L, Capote F, Teran J, Masa JF, Alonso ML, Corral J, Sánchez-Armengod A, Martinez C, Barceló A, Gozal D, Marín JM, Barbé F (2008) Daytime sleepiness and polysomnography in obstructive sleep apnea patients. Sleep Med 9(7):727-731

24. Häusler, N., et al., Association of napping with incident cardiovascular events in a prospective cohort study. Heart, 2019: p. heartjnl2019-314999

25. MedUni Vienna. 2019 [cited 2019 15-05]; Available from: https:// www.meduniwien.ac.at/web/ueber-uns/news/detailseite/2018/ news-im-maerz-2018/oesterreicher-leiden-haeufiger-anschlafproblemen-als-frueher/

26. Mezick EJ et al (2008) Influence of race and socioeconomic status on sleep: Pittsburgh SleepSCORE project. Psychosom Med 70(4): 410-416

27. Ibrahim AK, Kelly SJ, Adams CE, Glazebrook C (2013) A systematic review of studies of depression prevalence in university students. J Psychiatr Res 47(3):391-400

28. Chang A-M et al (2015) Evening use of light-emitting eReaders negatively affects sleep, circadian timing, and next-morning alertness. Proc Natl Acad Sci 112(4):1232-1237

29. Chellappa SL, Steiner R, Oelhafen P, Lang D, Götz T, Krebs J, Cajochen C (2013) Acute exposure to evening blue-enriched light impacts on human sleep. J Sleep Res 22(5):573-580

30. Blume, C., C. Garbazza, and M. Spitschan, Effects of light on human circadian rhythms, sleep and mood. Somnologie, 2019: p. 110

31. European Commission. Summertime consultation: $84 \%$ want Europe to stop changing the clock 2019 [cited 2019 15-05]; Available from: https://ec.europa.eu/transport/themes/ summertime/news/2018-08-31-consultation-outcome en

32. Buhr ED, Yoo S-H, Takahashi JS (2010) Temperature as a universal resetting cue for mammalian circadian oscillators. Science 330(6002):379-385

33. Riemann D et al (2017) S3-Leitlinie nicht erholsamer schlaf/ schlafstörungen. Somnologie 21(1):2-44

Publisher's note Springer Nature remains neutral with regard to jurisdictional claims in published maps and institutional affiliations. 